\title{
Electrical And Electrochemical Characteristics of Withania Somnifera Leaf Extract Incorporation Sodium Alginate Polymer Film For Energy Storage Applications
}

\section{K. Chinnaiah}

International Research centre

\section{T. Theivashanthi}

International Research centre

Karthik Kannan

Kumoh National Institute of Technology

\section{M.S. Revathy}

International Research centre

\section{Hemalatha Parangusan}

Centre for Research and P.G. Department of Physics

\section{S. Christopher Jeyaseelan}

PG \& Research Department of Physics

K. Gurushankar ( $\square$ gurushankar01051987@gmail.com )

South Ural State University https://orcid.org/0000-0003-1173-2859

\section{Research Article}

Keywords: AC electrical properties, Withania somnifera, sodium alginate, electrochemical window, supercapacitor

Posted Date: August 31st, 2021

DOI: https://doi.org/10.21203/rs.3.rs-826185/v1

License: (c) (1) This work is licensed under a Creative Commons Attribution 4.0 International License. Read Full License

Version of Record: A version of this preprint was published at Journal of Inorganic and Organometallic Polymers and Materials on November 10th, 2021. See the published version at https://doi.org/10.1007/s10904-021-02139-2. 


\section{Abstract}

The current investigation address the Alternating current (AC) electrical properties, electrochemical characteristics on biopolymer as Withania somnifera leaf extract incorporated sodium alginate polymer film prepared by solution casting method. Some crystallinity along with amorphous nature of sodium alginate emerged due to the incorporation of Withania somnifera leaf extract. The more amount of strain occurred in crystallization provides broaden peak in X-ray Diffraction (XRD) pattern. With aid of guluronic and mannuronic acids in sodium alginate making hydroxyl bond formation with leaf extract due to the cross linking process can be analyzed from Fourier transform Infrared Spectroscopy (FTIR). At room temperature, the prepared $5 \mathrm{ml}$ of leaf extract incorporated sodium alginate film has demonstrated highest ionic conductivity in the typical value of $2.80 \times 10^{-6} \mathrm{~S} / \mathrm{Cm}$. The electrochemical window of the prepared polymer film in the range of -0.4 to $0.4 \mathrm{~V}$ suggesting that used as an electrolyte for supercapacitor applications.

\section{Introduction}

Polymers are long-chain monomers and it consisting of complex network form, which offers the understood of the unrealized macroscopic behaviour of molecules in an interdisciplinary system [1]. Naturally derived biopolymers from living organism such as plant, trees, algae are well suitable for biological and pharmaceutical industries because of that having biodegradable and biocompatible characteristics [2]. The huge amount of availability as natural resources and reproducibility of alginate may consider as a promising material in biodegradable and edible films. Alginate is a class of natural biopolymer, which has hydrophilicity, non-toxic, soluble in water, anionic, and low cost in nature. This flexibility of alginate is making as a base material in various technological applications such as food, drug delivery, and cosmetics areas [3]. Generally, alginates are form of polysaccharides that are derived from brown seaweed algae, they chemically consisted of linear, unbranched polysaccharide monomers of 1-4 linked a-L-guluronic, and $\beta$-D-mannuronic acid residues are blocking on alternatively. It will form as homopolymeric (MM- or GG blocks) or heteropolymeric (MG- or GM block) sequences. Even structures are produced by $\mathrm{M}$ blocks, the egg-box type structures formed by G-block and more flexible structures are developed by MG block [4]. The crosslinking network of alginate film is easily thinkable because it possesses a large number of hydroxyl groups, and extra oxygen as well as making bond formation with any other cations [5]. Withania somnifera is an important reverted herb in Indian Ayurveda medicines their various aerial parts are used in various biological activities. Murad Ali Khan et.al have reported Withania somnifera having various metals like chromium, lead, copper, cadmium, nickel, manganese, and Iron [6]. The conductive polymers are considered electrode materials nowadays due to their fast ion/electron transfer process and specific surface areas. The high ionic conductivity of polymer electrolytes offers various advantages like flexibility, processability, and enhanced resistance to variations in the charge/ discharge process. In addition, biopolymer electrolytes have good chemical, thermal, and mechanical properties compared to synthetic hence they will be used for replacing synthetic polymer in electrochemical devices. Jothi et.al has used corn starch in proton-conducting polymer investigations [7]. 
Generally, the energy storage devices such as supercapacitor, batteries, electrochemical solar cells, electrochromic cells, and fuel cells are characterizing by solid state ionics and electrochemical investigations [8]. Hence, the present work aims to investigate the ionic conductivity and electrochemical performance of the sodium alginate film using Withania somnifera leaf extract by solvent casting method. The incorporated Withania somnifera leaf extract on sodium alginate polymer film is improved their ionic conductivity from $1.24 \times 10^{-7}$ to $2.80 \times 10^{-6} \mathrm{~S} / \mathrm{cm}$. Previous literature are shown similar kind of ionic conductivity in the development of biopolymer-based membrane by adding inorganic or synthetic polymers for eg. Sadrabadi et.al have shown $1.67 \times 10^{-6} \mathrm{~S} / \mathrm{cm}$ for double layer chitosan and Yuwei Zhang et.al have shown $2.2 \times 10^{-6} \mathrm{~S} / \mathrm{cm}$ for chitosan-PVA/Nafion and Sunder et.al have reported $1.59 \times 10^{-6}$ $\mathrm{S} / \mathrm{cm}$ for lithium bromide PEO/PVP electrolyte [9-11]. However, in this work ionic conductivity improved through the incorporation of leaf extract without blending of other polymers, and adding of any other salt.

\section{Experimental}

\subsection{Materials}

The homogeneous Withania somnifera leaves are collected from hills station of Kodaikanal, Tamilnadu, India, and sodium alginate is purchased from SRL chemicals, Mumbai, India used without further purifications for the preparation of sodium alginate polymer film.

\subsection{Preparation of sodium alginate polymer film incorporated by Withania somnifera leaf extract}

The Withania somnifera leaf extract incorporated sodium alginate polymer film is synthesized via the ordinary casting method. $10 \mathrm{~g}$ of fresh Withania somnifera leaves cutting on uniformly then soaked in $100 \mathrm{ml}$ of double distilled water in $250 \mathrm{ml}$ conical flask and stirred with $70^{\circ} \mathrm{C}$ for 1 hour. The obtained solution was cool to room temperature and filtered by Whatman No.1 filter paper. The pure filtered extract was used to further process. The $0.5 \mathrm{~g}$ of sodium alginate is dissolved in $50 \mathrm{ml}$ of water and this mixture is stirred constantly for 1 hour at $70^{\circ} \mathrm{C}$. The complete yellow colour solution formed after the complete dissolution of sodium alginate. Now, various wt. \% like $2.5 \mathrm{ml}, 5 \mathrm{ml}$, and $10 \mathrm{ml}$ of Withania somnifera leaf extract is added to the yellow colour sodium alginate solution. These solutions are stirred continuously for up to $24 \mathrm{hrs}$, to get a consistent viscous solution. The same procedure was used for the preparation of pure sodium alginate film. The gathered viscous solution was poured into a polypropylene petri dish and dried for five days at room temperature. Free-standing films with an average thickness range of $0.06 \mathrm{~cm}$ have been obtained. These films are subject to studies of various characterizations such as Fourier Transform Infrared spectroscopy (FTIR), X-ray diffraction analysis (XRD), Impedance analysis, and cyclic voltammetry (CV).

\subsection{Characterization techniques}

The prepared Withania somnifera leaf extract incorporated sodium alginate polymer film Structural behaviour was analyzed through X-ray diffractometer [Brucker Eco D8 Advance with Cu-Ka radiation ( $\lambda=$ 
$1.54060 \AA$ ) ] with a scanning rate of $5^{\circ} /$ minute in the range of $10-60^{\circ}$. Functional groups presenting on polymer matrix are identified through FTIR spectrum carried out using IRTracer-100 Shimadzu with spectrum range from $4000-400 \mathrm{~cm}^{-1}$ using $\mathrm{KBr}$ as a reference at room temperature. The electrochemical impedance spectroscopy (EIS) of the prepared polymer film was performed on HIOKI 3532-50 LCR HI-TESTER with a frequency of $1 \mathrm{kHz}$. The electrochemical analysis of prepared alginate film was carried out in electrochemical instrument, model- CHI60008E, USA).

\section{Results And Discussion}

\subsection{FTIR analysis}

The FTIR spectra have been used to investigate the occurred chemical composition and their interactions in the prepared sample. Figure 1a displays FTIR spectra of pure and Withania somnifera leaf extract incorporated sodium alginate polymer film. Both spectra show a broad-band centered absorption at approximately $3300 \mathrm{~cm}^{-1}$ this may be arises from the stretching mode of the $\mathrm{OH}$ hydroxyl group of phenols and alcohols and the low-intensity peak at $2920 \mathrm{~cm}^{-1}$ indicates $-\mathrm{CH}_{2}$ group of alkyls. The peaks appeared at 1589 and $1413 \mathrm{~cm}^{-1}$ are attributed to symmetric and asymmetric mode of carboxylate $\left(\mathrm{COO}^{-}\right)$salt group and peak at $1323 \mathrm{~cm}^{-1}$ shows plane deformation of the $\mathrm{C}-\mathrm{H}$ group. The number absorption band assigned at 1105,1060 , and $1028 \mathrm{~cm}^{-1}$ reveal the existence of $\mathrm{C}-0-\mathrm{C}$ glycoside bonds in polysaccharides [12]. The peaks are observed at $945,896,813,715$, and $615 \mathrm{~cm}^{-1}$ suggested the presence of guluronic acid and mannuronic acids in sodium alginate [13]. The Withania somnifera leaf extract incorporated sodium alginate FTIR spectra in Fig. 1b shows the disappearance of peak at 945 $\mathrm{cm}^{-1}$ which confirms that guluronic acid from sodium alginate strongly binder with Withania somnifera leaf extract. In addition, reduction of intensity of carboxylate salt group at $1589 \mathrm{~cm}^{-1}$ reveals the crosslinking of sodium alginate with leaf extract through the ionic bonding. This is assured by the formation of a new peak at 2204, 2152, and $2036 \mathrm{~cm}^{-1}$ which are belong to ionic bonding of $\mathrm{OH}$ hydroxyl groups, hence to be obtained homogeneously dispersed solution during synthesis.

\subsection{XRD analysis}

The crystallinity/amorphous nature of pure and leaf extract incorporated sodium alginate film are determined through XRD analysis and their diffraction pattern is shown in Fig. 2. As shown in Fig. 2a, Two characteristic broad humps can be seen from $10-30^{\circ}$ are illustrating the semicrystalline nature of sodium alginate because of ions dislocations occurred in the prepared sample, it is a well agreement with earlier reports [7]. The appearance of crystalline peaks at $31.44^{\circ}, 32.53^{\circ}$, and $56.14^{\circ}$ in Fig. $2 \mathrm{~b}$ is due to the addition of leaf extract, and it has been introduced some crystallinity along with amorphous nature. The observed crystalline peaks are due to the intermolecular interaction between the hydroxyl groups and leaf extract, which are helpful to the formation of hydrogen bonds and facilitate the chain movement of sodium alginate [14]. The increasing crystallinity exhibits the saturation state of the host matrix and this may be due to the recombination of ions. Furthermore, it has been indicated incomplete dissociation of 
leaf extract in sodium alginate through the recrystallization process while adding on leaf extract. These characters are may restrict the motion of ions in the electric field [15]. Thus from XRD data, we can understand sodium alginate well connected to Withania somnifera leaf extract via ionic bonding.

The crystallite size of recrystallized sodium alginate has been calculated from Debye-Scherer's formula

$D=k \lambda / \beta \cos \theta-(1)$

Where; $D$ represents the crystalline size, $k$ represents Scherer constant $(k=0.94), \lambda$ represents $X$-ray wavelength $\left(\lambda=1.54178 \mathrm{~A}^{\circ}\right)$ for $\mathrm{Cu} \mathrm{K}_{\mathrm{a}}$ radiation, $\beta$ represents full width half maximum (FWHM) and $\theta$ represents diffraction angle. The calculated average peak crystallite size is found to be $22 \mathrm{~nm}$.

Furthermore, this crystallite size was again evaluated by stress-strain plot (Williamson - Hall) method displayed in Fig. 3. The graph is plotted between $4 \sin \theta V s \beta \cos \theta$ and fitted on linear fitting origin lab software. This figure represents recrystallized sodium alginate strain is assumed to be uniformly in all crystallographic directions, thus considered the isotropic nature of crystal. According to the W-H method

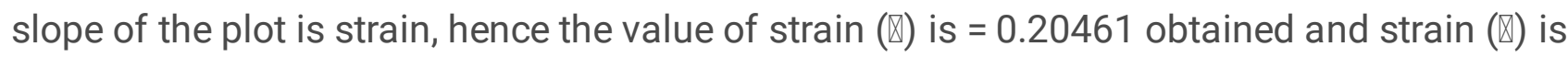
dimensionless quantity because of the ratio between the two lengths. The value of $y$-axis - intercept " $c$ " = 0.156 is used to calculate the crystallite size and calculated from the following equation

$[Y-$ Intercept " $\mathrm{C}$ " $=\mathrm{K} \lambda / \mathrm{D}$ and $\mathrm{K} \lambda / 0.156=\mathrm{D}]-(2)$

The calculated crystallite size is $8.8 \mathrm{~nm}$ by the stress-strain plot method. The crystallite size of Scherer's method is two times more than the crystallite size of the stress-strain method. The strain is more nonuniform in formed sodium alginate film and this behaviour exhibit broadened peak in XRD analysis. A similar kind of result is discussed by earlier literature [16].

\subsection{Impedance analysis}

The pure sodium alginate and Withania somnifera leaf extract incorporated sodium alginate polymer Cole-Cole plots at different temperatures are shown in Fig. 4. The different wt\% of Withania somnifera leaf extract incorporated sodium alginate Cole-Cole plots is shown in Fig. 4a. In our study, all the graphs are shown the semi-circular portion in one spike. The semicircle on Cole-Cole plot in high-frequency regions illustrates the parallel combination of bulk resistance and bulk capacitance present on polymer because of its found to be in bulk effect in the electrolyte. The spike on Cole-Cole plot in low-frequency region shows that the blocking effect to be obtained on electrode/electrode interface [17]. The ionic conductivity of the prepared polymer is calculated from the following formula

$\left[\sigma=l /\left(R_{b} A\right)\right] s / c m-(3)$

Where, I represents thickness $\left(\mathrm{cm}^{2}\right)$ of alginate film, A indicates the surface area $\left(\mathrm{cm}^{2}\right)$ of the alginate film and $R_{b}$ represents the bulk resistance (ohms) of alginate film. The bulk resistance $\left(R_{b}\right)$ of polymer is intercepting on the real axis at the lower frequency of the end of the Cole-Cole plot, which has been calculated by Z view software. The pure sodium alginate Cole-Cole plots are showing the different bulk 
resistance values for different temperatures. While increasing the temperature from the ambient condition, the bulk resistance increases up to level of $358 \mathrm{~K}$ after this temperature alginate film has been started to exhibits decreased bulk resistance and it has lies between 328-348 K. The pure sodium

alginate film exhibits the highest ionic conductivity at $1.24 \times 10^{-7} \mathrm{~S} / \mathrm{cm}$. As the concentration of Withania somnifera leaf extract changes from 2.5 up to $10 \mathrm{ml}$ also showing some degree of changes in Cole-Cole plot. While changing wt. \% of Withania sominefera leaf extract, $5 \mathrm{ml}$ of leaf extract incorporated film only showing the lowest bulk resistance and improved ionic conductivity at room temperature when compared to others such as pure sodium alginate, $2.5 \mathrm{ml}$, and $10 \mathrm{ml}$ of leaf extract incorporated film. Hence, we are used $5 \mathrm{ml}$ of leaf extract only used to further studies of various impedance spectra at different temperatures and electrochemical analysis. The improved ionic conductivity of $5 \mathrm{ml}$ leaf extract's incorporated alginate film is $1.24 \times 10^{-7} \mathrm{~S} / \mathrm{cm}$ which is due to the number of free ions migration in the alginate polymer film is increased by the addition of leaf extract. The leaf extract's other concentrations are making more crystallinity in polymer electrolyte.

\subsection{Conductance spectra analysis}

The conductivity of pure sodium alginate and Withania somnifera leaf extract incorporated polymer film were determined from the following relation

$[\sigma=n e \mu]-(4)$

Where $\mathrm{n}$ and $\mu$ are the number of charge carriers, mobility of the polymer respectively. Figure 5 depicts the plot of conductivity log $\left(\sigma_{a c}\right)$ vs log (f) for various temperatures of the optimized and pure alginate polymer film. The plots are exhibit all the three well-defined conductance spectra religions. The lowfrequency dispersion region to be obtained with low conductivity value due to the space charge polarisation or interfacial impedance occurred in the blocking electrode which indicates the Non-Debye characteristics of studied polymer film [18]. The middle frequency-independent plateau region shows the DC conductivity of bulk material, which is due to the migrations of ions to the neighbouring side. The DC conductivity value calculated from the conduction spectra by extrapolating the middle frequencyindependent plateau region meets on log $\sigma$ in y-axis. The maximum DC conductivity obtained for $358 \mathrm{~K}$ typically their value is $1.98 \times 10^{-5}$. This is a good agreement with the Cole-Cole plot. At higher frequency region, conductivity $(\sigma)$ increases with increasing $\log (f)$. Moreover, the conductivity value of leaf extract incorporated polymer film was found to be higher compared to pure sodium alginate polymer which implies that leaf extract incorporation within the polymer may augment the mechanism of charge conduction moderately faster. Also, this augmentation may note out to increase in disorder degree that controls the mobility of charge carriers and reveals the creation of an interconnected percolating chain that is appropriate for the mechanism of charge transfer. The increasing conductivity of leaf extract incorporated alginate polymer represents leaf extract is suitable for the synthesis of sodium alginate polymer film for electrolyte applications.

\subsection{Dielectric analysis}


Figure 6 displays the variation of the real part of complex permittivity $\left(\nabla^{\prime}\right)$ for pure sodium alginate and leaf extract mediate studied film for different temperatures. The permittivity ( $\left.\mathbb{\Xi}^{\prime}\right)$ of pure and leaf extract incorporated studied films are shown the frequency and temperature dependence. It has been exhibited decreasing permittivity with increasing temperature and becomes saturated at high-frequency levels. The high dielectric permittivity of both films are obtained at lower frequencies might be on grounds that indicates dipoles have enough time to make changes in the electric field [19]. The dielectric permittivity scaling down is due to the inability of dipoles to align rapidly in the electric fields at high frequencies. The increasing real dielectric permittivity may be due to the decrement of charge carrier in sodium alginate which can be attributed to the homogeneous distribution of leaf extract in sodium alginate film. The accumulation of charge carriers at internal interfaces of studied films is giving information about the micro-capacitor network which is demonstrated by Maxwell-Wagner-sillar affects [20]. At low frequencies, real dielectric permittivity increases with increasing of temperature may be due to the slight decreasing of sodium alginate viscosity and this behaviour making easy rotation of dipoles with AC electric field, which are causing the molecular orientation and rearrangement increment with temperature [21]. The interfacial and dipolar polarization is temperature-dependent that is creates a significant effect on the dielectric constant of leaf extract incorporated alginate film. The combined effects of interfacial and dipolar polarization are resulting in a rapid increment of dielectric constant with the temperature at lower frequencies.

The imaginary part of complex permittivity $\left(\nabla^{\prime \prime}\right)$ of pure and Withania somnifera leaf extract incorporated polymer film at different temperature are shown in Fig. 7a and 7b. It was displayed that temperature dependence at a lower frequency. The imaginary part of complex permittivity ( $\left.\nabla^{\prime \prime}\right)$ increases while increasing the temperature from ambient to $358 \mathrm{~K}$ at lower frequencies. The influence of temperature not making any changes at high frequency, as predicted which could be interpreted by the suggested model of Maxwell-Wagner.

\subsection{Modulus spectra analysis}

Figure 8 and Fig. 9 depicts the real and imaginary part of the dielectric modulus of pure and leaf extract incorporated sodium alginate polymer film. At lower frequencies, the values of $\mathrm{M}^{\prime}$ and $\mathrm{M}^{\prime \prime}$ are close to zero for both films at different temperatures. The electrode and electrolyte interface effects of $\mathrm{M}^{\prime}$ and $\mathrm{M}^{\prime \prime}$ contributions are negligible in this region. Hence, it was exhibited a long-tail appearance of a certain frequency and having a large capacitance related to the electrodes. However, it indicates that non-Debye nature of prepared samples. While on high frequencies, the values of $\mathrm{M}^{\prime}$ increase for all temperatures and reach the optimum value. This may be caused by a lack of restoring forces to the carrier charges under in theaction of the motivated applied field. These kinds of behaviour have been obtained in many sodium alginate polymers [22]. The peaks of $\mathrm{M}$ " are represented the relaxation conductivity of mobile ions and are related to the dynamics of transition ions. The values of $\mathrm{M}$ " decrease with increasing temperature and peaks are moving towards the high frequencies assure that the relaxation mechanism having a polarity. The shift of the $\mathrm{M}^{\prime \prime}$ relaxation peaks gives information about the incorporation of leaf extract that may reduce the interfacial strength of the polymeric matrix. Argand et al. plot real $\left(M^{\prime}\right)$ and imaginary $\left(M^{\prime \prime}\right)$ to 
display the relaxation process nature of leaf extract incorporated polymer film in Fig. 10. It shows semicircular formation, which depicts the Debye model relaxation process, single relaxation time in the studied film [23]. In this model, all the dipoles might be identical and this ideal dipole relaxation to the alternating electric field makes the Debye model.

\subsection{Tangent analysis}

The variation of dielectric dissipation factor tan $\delta$ with a frequency of leaf extract incorporated sodium alginate polymer film is shown in Fig. 11. The tangent analysis is used to analysing the relaxation mechanism of the prepared samples. It denotes ratio between the amount of energy loss and the amount stored in a polymer film. Generally, that has been calculated from the following equation.

$\left[\tan \delta=\left(\nabla^{\prime \prime} / \mathbb{\bigotimes}^{\prime}\right)\right]-(5)$

The $\tan \delta$ values are to increase with frequency at different temperatures attains maximum than decreases further increasing of frequencies. When increasing the temperature $\tan \delta$ frequencies are shifted towards lower frequency, this behaviour ensures that studied polymer films have parallel RC elements. This frequency shifting is established by the increment of charge carrier density in this sample; hence, the addition of leaf extract makes faster segmental relaxation in this sample.

\subsection{Electrochemical analysis}

\subsubsection{Cyclic voltammetry}

The electrochemical reversibility performance of the prepared polymer is analyzed by cyclic voltammetry. The measurement of $\mathrm{CV}$ analysis has been carried by silver||electrolyte\|silver configuration. This plot has taken on potential window $-0.4 \mathrm{~V}$ to $0.4 \mathrm{~V}$ at ambient temperature with various scan rates such as 25,20 , 15,10 , and $5 \mathrm{mVs}^{-1}$ are as shown in Fig. 12. It shows that well defined anodic and cathodic current peaks, which are pointed out the pseudo capacitor behaviour of the prepared sample. The fast ionic reactions at the nearby surface region of the electrode produce this pseudo capacitor behaviour due to oxidation and reduction reactions are occurred at faradic energy storage system [24]. The linearly increased titled spark in Cole-Cole plots at low-frequency regions are proved this capacitive nature, which is occurred by the ionic absorption at the electrode-electrolyte interface. The redox peaks are increasing while increasing on scan rate, which is caused by the increment of ions migration due to decreased resistance. Hence, improve the accumulation of charges at electrode-electrolyte boundary. The simultaneous broadening area of the CV curve along with scan rate confirms the interfacial compatibility, polaron, and ionic conduction in prepared Withania somnifera leaf extract incorporated sodium alginate polymer sample.

\section{Conclusion}

In summary of this report, we have prepared sodium alginate polymer electrolyte through the incorporation of different concentrations Withania somnifera leaf extract by the solution casting method. 
This leaf extract incorporated sodium alginate electrolyte exhibits the improved maximum conductivity $2.80 \times 10^{-6}$ from $1.24 \times 10^{-7} \mathrm{~S} / \mathrm{cm}$ at room temperature through the regulation of an appropriate amount of ionic liquid. This increasing conductivity represents Withania somnifera leaf extract is suitable for the synthesis of sodium alginate polymer film. While increasing the temperature of prepared polymer film giving the following results i) bulk resistances are increases up to $358 \mathrm{~K}$ ii) maximum DC conductivity $1.98 \times 10^{-5}$ obtained on $358 \mathrm{~K} \mathrm{iii)} \mathrm{Real} \mathrm{dielectric} \mathrm{permittivity} \mathrm{increases} \mathrm{with} \mathrm{increasing} \mathrm{of} \mathrm{temperature} \mathrm{that}$ making easy rotation of dipoles with AC electric field. Hence, rapid increment of dielectric constant with temperature given by combined effect of interfacial and dipolar polarization. The XRD analysis reveals that incorporation of leaf extract introduces some crystalline nature along the amorphous of sodium alginate due to recrystallization process by itself found on dislocations in sodium alginate. Thus, results are facilitated chain movement through the formation of hydrogen bonding and show the well and good bind of leaf extract with sodium alginate. Further, the results were supported and confirmed by the disappearance of glucuronic acid peaks in FTIR spectra by the ionic formation via cross-linking of leaf extract to sodium alginate. The recrystallized sodium alginate crystal nature evaluated by WilliamsonHall method, its demonstrated their crystallographic directions is isotropic. The cyclic voltammetry analysis confirmed well-defined oxidation and reduction peaks at potential window ranges of $-0.4 \mathrm{~V}$ to 0.4 $V$ and their pseudo capacitive nature was proved in the Cole-Cole plot at low-frequency region by the linearly increased titled spark. Based on the results it suggests that Withania somnifera leaf extract mediated sodium alginate electrolyte is one the promising candidate for supercapacitor application.

\section{Declarations}

Author Contributions KC TT, KG and MSR were involved in the preparation of sodium alginate polymer film incorporated by Withania somnifera leaf extract, and the preparation of the manuscript. KK, VK, HP and SC helped in the characterization techniques and drafting the manuscript. KG, KK, and VK revised the manuscript, KC analysed the data and revised the manuscript, KG drafted and revised the manuscript. All the authors read and approved the fnal manuscript.

Declarations Conflict of interest Authors declare that there is no conflict of interest among the co-authors.

\section{References}

1. A. Ciferri, Translation of molecular order to the macroscopic level. Chem. Rev. 116, 1353-1374 (2016).

2. S. De, D. Robinson, Polymer relationships during preparation of chitosan-alginate and poly-Hysinealginate nanospheres. J. Control. Release 89, 101-112 (2003).

3. C. G. Gomez, M. Rinaudo, M. A. Villar, Oxidation of sodium alginate and characterization of the oxidized derivatives. Carbohydr. Polym. 67, 296-304 (2007).

4. H. Daemi, M. Barikani, Synthesis and characterization of calcium alginate nanoparticles, sodium homopolymannuronate salt and its calcium nanoparticles. Sci. Iran. F 19, 2023-2028 (2012). 
5. N. K. Sachan, S. Pushkar, A. Jha, A. Bhattcharya, Sodium alginate: the wonder polymer for controlled drug delivery. J. Pharm. Res. 2, 1191-1199 (2009).

6. M. A. Khan, I. Ahmad, I. U. Rahman, Effect of Environmental Pollution on Heavy Metals Content of Withania somnifera. J Chin Che Soc. 54, 339-343 (2007).

7. M. A. Jothi, D. Vanitha, N. Nallamuthu, A. Manikandan, S. Asath Bahadur, Investigations of lithium ion conducting polymer blend electrolytes using biodegradable cornstarch and PVP. Physica B Condens. Matter 580, 411940 (2020).

8. K. Jeyabanu, K. Sundaramahalingam, P. Devendran, A. Manikandan, N. Nallamuthu, Effect of electrical conductivity studies for CuS nanofillers mixed magnesium ion based PVA-PVP blend polymer solid electrolyte. Physica B Condens. Matter 572, 129-138 (2019).

9. M. M. Hasani-Sadrabadi, E. Dashtimoghadam, F. S. Majedi, S. H. Emami, H. Moaddel, A highperformance chitosan-based double layer proton exchange membrane with reduced methanol crossover. Int. J. Hydrog Energy 36, 6105-6111 (2011).

10. Y. Zhang, Z. Cui, C. Liu, W. Xing, J. Zhang, Implantation of Nafion ${ }^{\circledR}$ ionomer into polyvinyl alcohol/chitosan composites to form novel proton-conducting membranes for direct methanol fuel cells. J. Power Sources 194, 730-736 (2009).

11. K. Sundaramahalingam, D. Vanitha, N. Nallamuthu, A. Manikandan, M. Muthuvinayagam, Studies on sodium nitrate based polyethylene oxide/polyvinyl pyrrolidone polymer blend electrolytes. Physica B Condens. Matter 547, 55-63 (2018).

12. M. Kuzmanović, D. K. Božanić, D. Milivojević, D. Mitić Ćulafić, S. Stanković, C. Ballesterose, J. Gonzalez-Benito, Sodium-alginate biopolymer as a template for the synthesis of nontoxic red emitting $\mathrm{Mn}^{2+}$-doped CdS nanoparticles. RSC Adv. 7, 53422-53432 (2017).

13. C. R. Badita, D. Aranghel, C. Burducea, P. Mereuta, Characterization of sodium alginate based films. Rom. J. Phys. 65, 602 (2020).

14. N. Shaari, S. K. Kamarudin, S. Basri, L. K. Shyuan, M. S. Masdar, D. Nordin, Enhanced mechanical flexibility and performance of sodium alginate polymer electrolyte bio-membrane for application in direct methanol fuel cell. J. Appl. Polym. Sci. 135, 46666 (2018).

15. S. Kiruthika, M. Malathi, S. Selvasekarapandian, K. Tamilarasan, V. Moniha, R. Manjuladevi, Ecofriendly biopolymer electrolyte, pectin with magnesium nitrate salt, for application in electrochemical devices. J Solid State Electrochem. 23, 2181-2193 (2019).

16. A. K. Zak, W. H. Abd. Majid, M. E. Abrishami, R. Yousefi, R. Parvizi, Synthesis, magnetic properties and $X$-ray analysis of $Z_{0.97} X_{0.03} \mathrm{O}$ nanoparticles $(X=M n, N i$, and $\mathrm{Co})$ using Scherrer and size-strain plot 
methods. Solid State Sci. 14, 488-494 (2012).

17. M. Premalatha, N. Vijaya, S. Selvasekarapandian, S. Selvalakshmi, Characterization of blend polymer PVA-PVP complexed with ammonium thiocyanate. Ionics 22, 1299-1310 (2016).

18. S. Ramesh, M. F. Chai, Conductivity, dielectric behavior and FTIR studies of high molecular weight poly(vinylchloride)-lithium triflate polymer electrolytes. Mater Sci Eng B 139, 240-245 (2007).

19. M. Amir, B. Ünal, M. Geleri, H. Güngünes, S. E. Shirsath, A. Baykal, Electrical properties and hyperfine interactions of boron doped $\mathrm{Fe}_{3} \mathrm{O}_{4}$ nanoparticles. Superlattices Microstruct. 88, 450-466 (2015).

20. Z.-M. Dang, L. Wang, Y. Yin, Q. Zhang, Q.-Q. Lei, Giant Dielectric Permittivities in functionalized carbonnanotube/electroactive-polymer nanocomposites. Adv. Mater 19, 852-857 (2007).

21. N. Singh, A. Agarwal, S. Sanghi, Dielectric relaxation, conductivity behavior and magnetic properties of Mg substituted Zn-Li ferrites. Curr. Appl. Phys. 11, 783-789 (2011).

22. S. Choudhary, Structural and dielectric properties of (PEO-PMMA)-SnO ${ }_{2}$ nanocomposites. Compos. Commun. 5, 54-63 (2017).

23. M. A. Morsi, A. H. Oraby, A. G. Elshahawy, R. M. Abd El-Hady, Preparation, structural analysis, morphological investigation and electrical properties of gold nanoparticles filled polyvinyl alcohol/carboxymethyl cellulose blend. J Mater Res Technol. 8, 5996-6010 (2019).

24. L. Xia, H. Huang, Z. Fan, D. Hu, D. Zhang, A. S. Khan, M. Usman, L. Pan, Hierarchical macro-/meso-/microporous oxygen-doped carbon derived from sodium alginate: A cost-effective biomass material for binder-free supercapacitors. Mater. Des. 182, 108048 (2019).

\section{Figures}




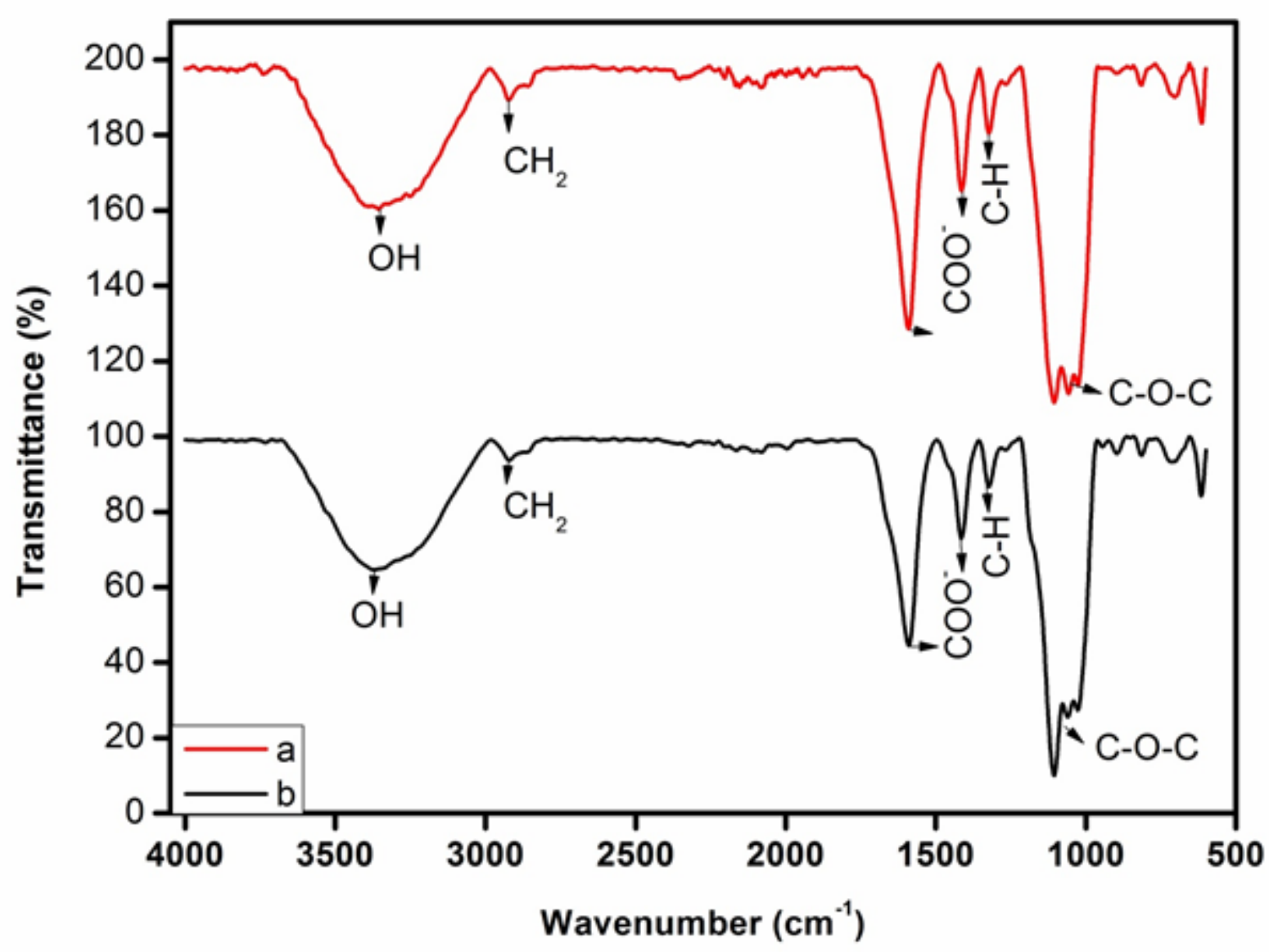

Figure 1

FTIR spectra of a) pure and b) Withania somnifera leaf extract incorporated sodium alginate polymer film 


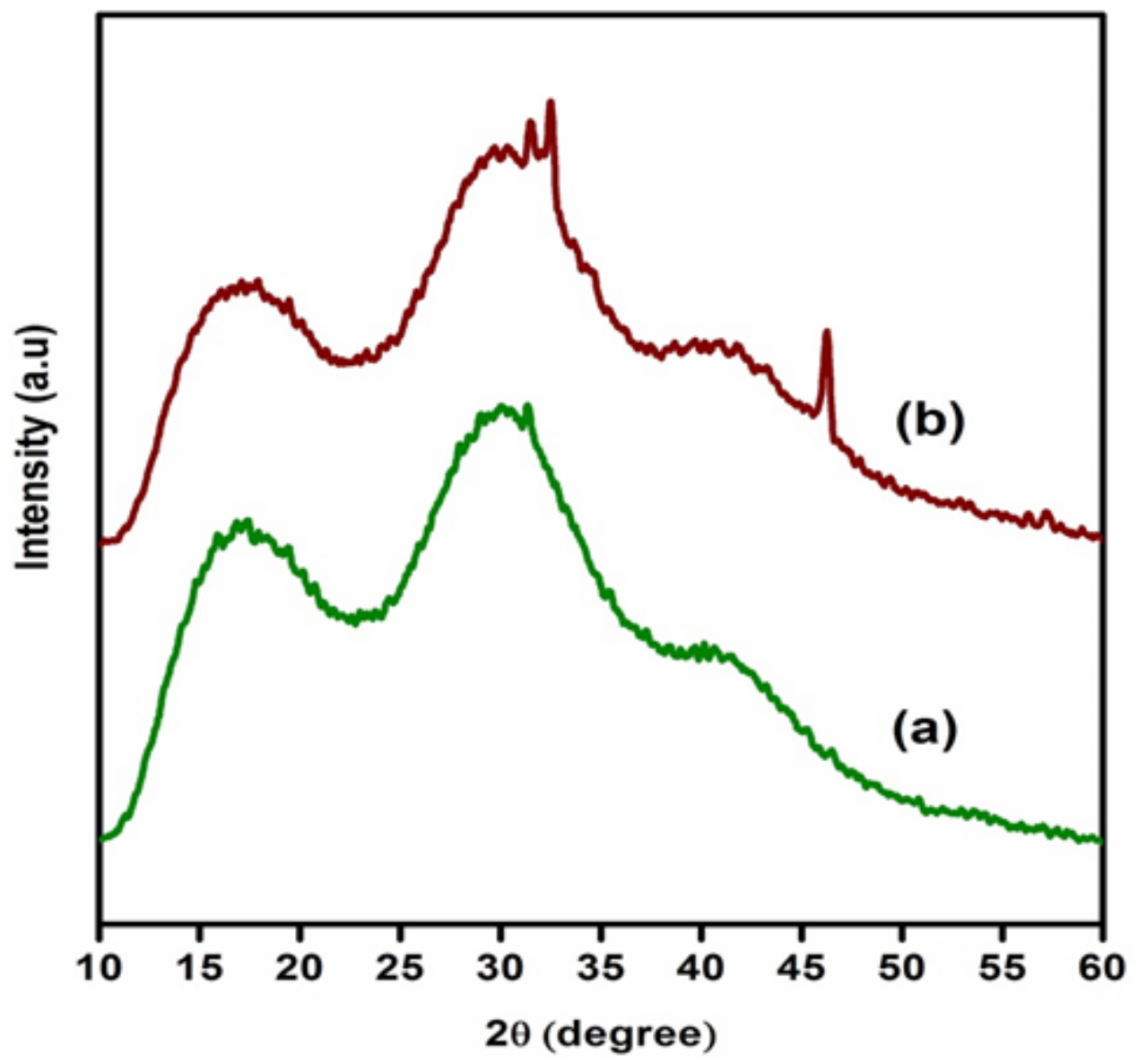

Figure 2

XRD pattern of a) pure and b) Withania somnifera leaf extract incorporated sodium alginate polymer film

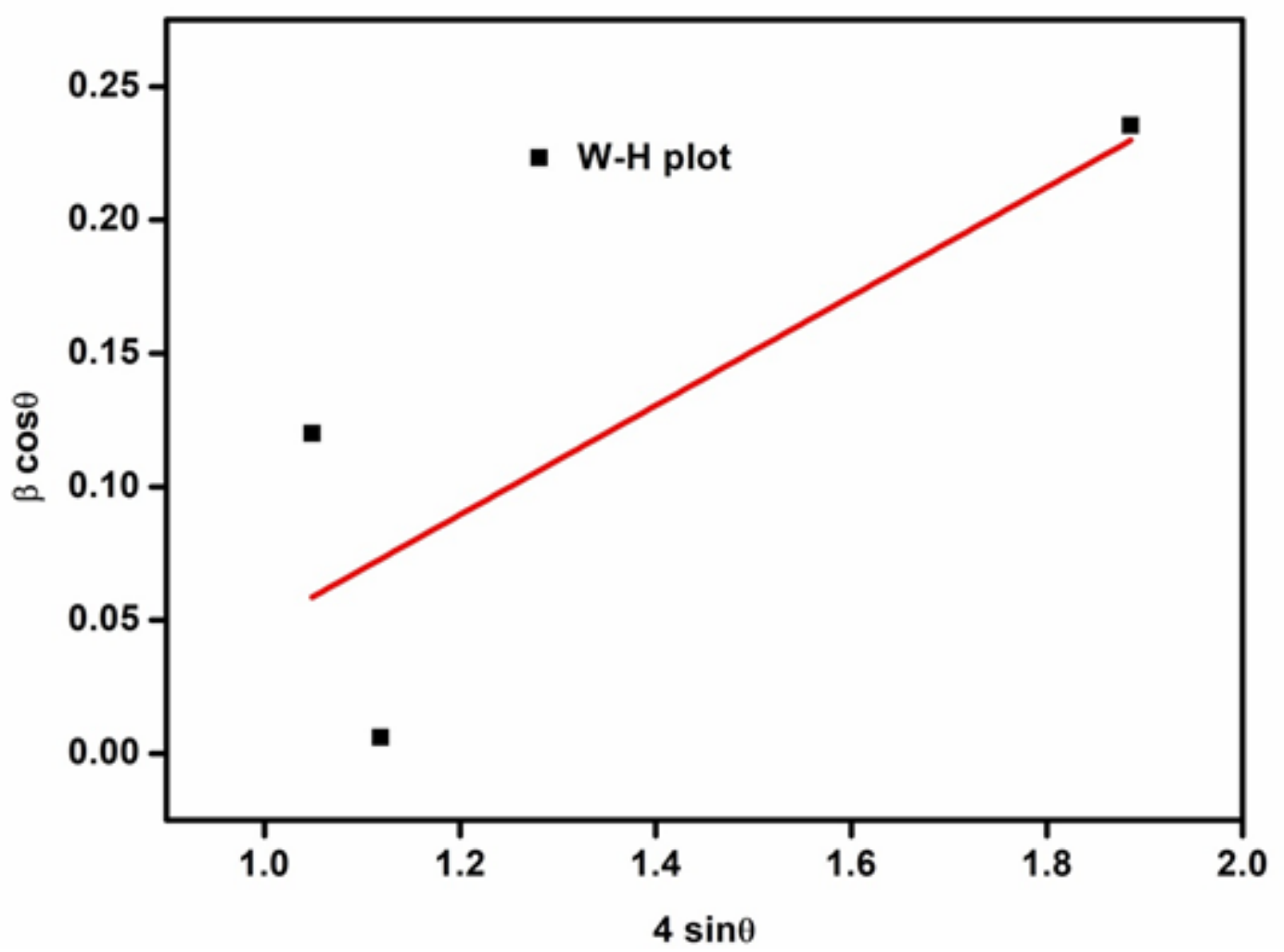




\section{Figure 3}

W-H plot of Withnia somnifera leaf extract incorporated sodium alginate polymer film
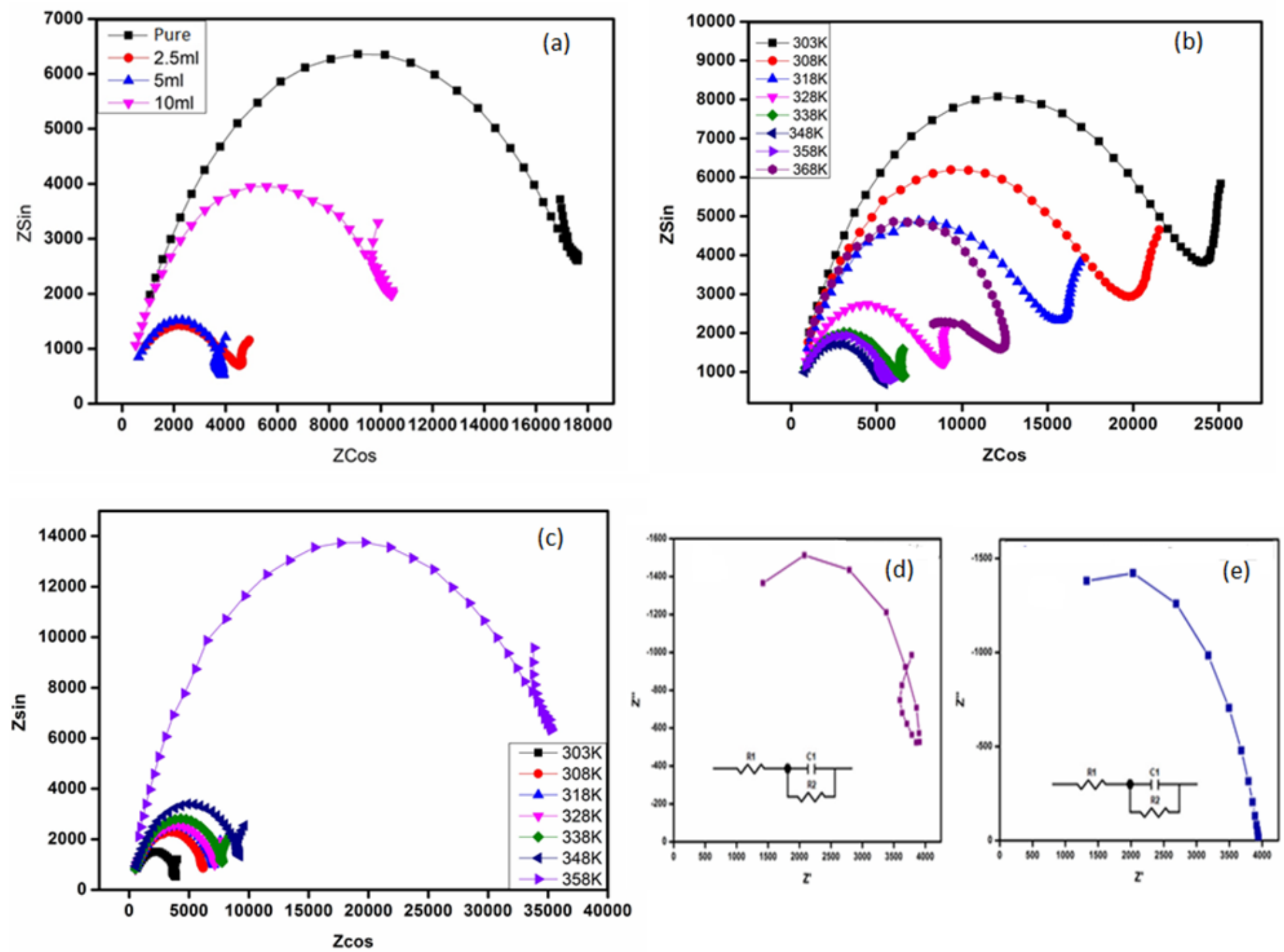

Figure 4

Cole-Cole Plot of pure and Withania somnifera leaf extract incorporated sodium alginate polymer film a) various concentration Withania somnifera leaf extract incorporation on sodium alginate at room temperature $b$ ) various temperature of pure sodium alginate $c$ ) optimised Withania somnifera leaf extract incorporated sodium alginate film at various temperature $(5 \mathrm{ml}) \mathrm{d}$ ) equivalent circuit of pure sodium alginate e) equivalent circuit of optimised sodium alginate polymer film 

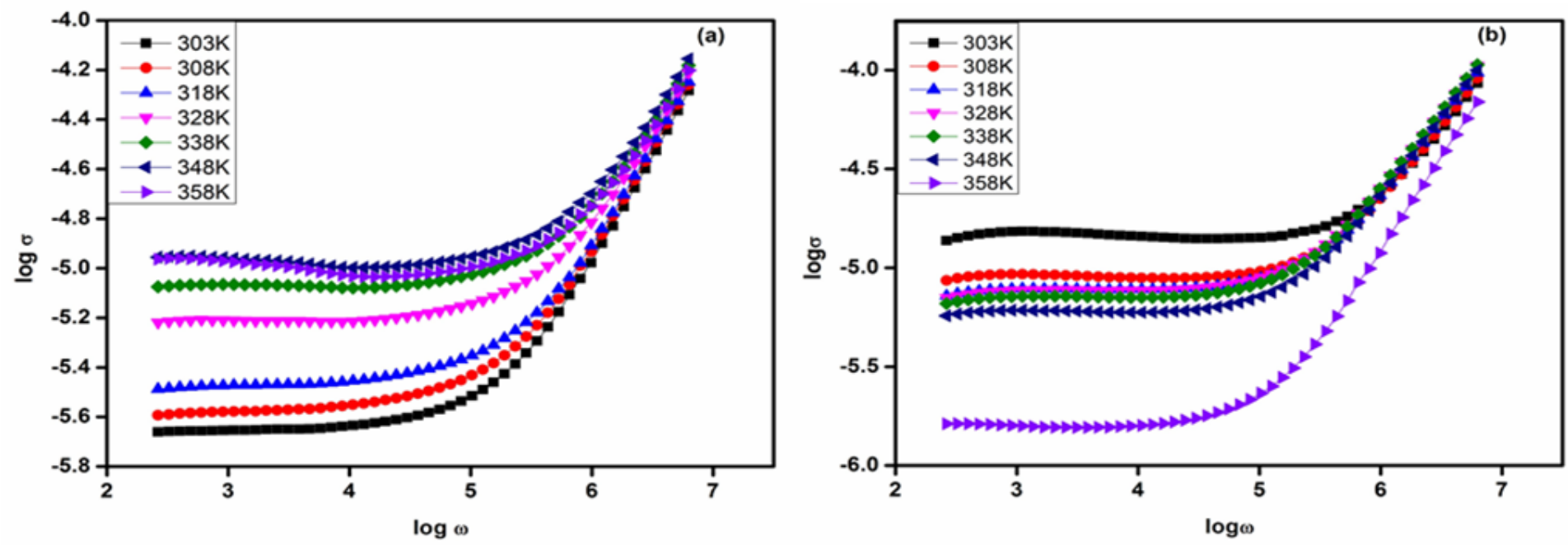

Figure 5

Conductance spectra of a) pure and b) Withania somnifera leaf extract incorporated sodium alginate polymer film at different temperatures
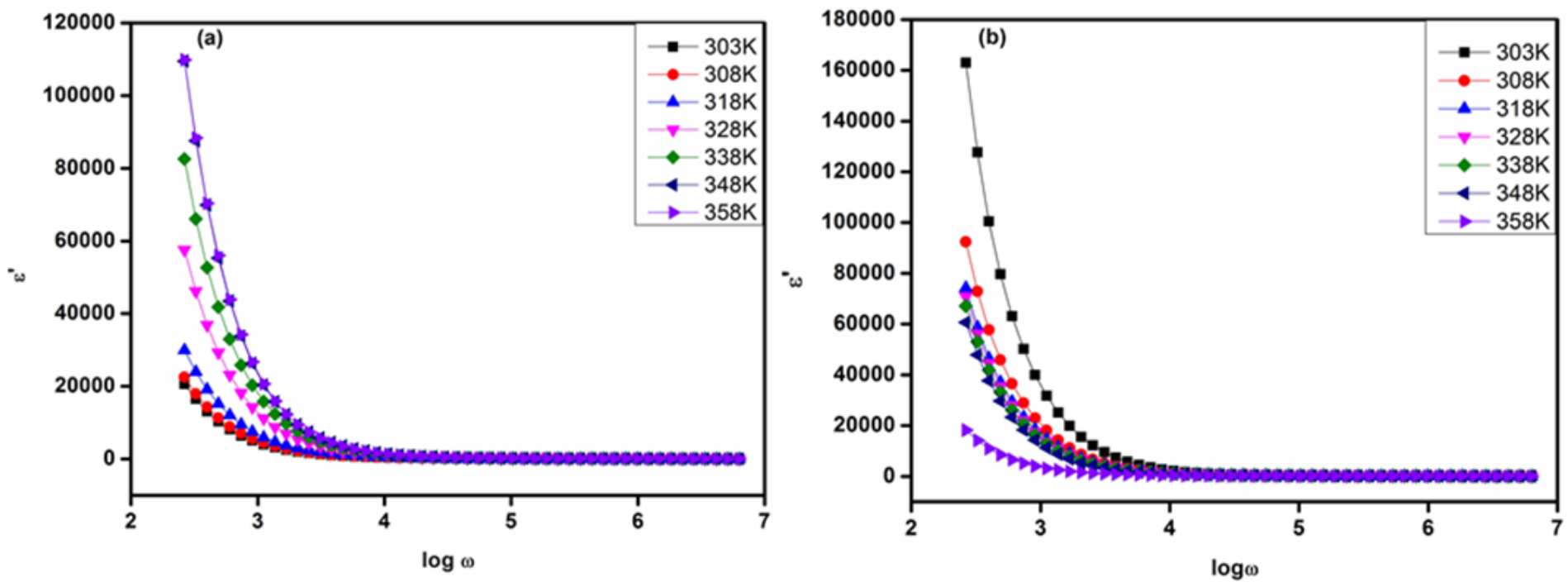

Figure 6

Real part of complex permittivity ( $\left.\mathbb{\Xi}^{\prime}\right)$ for a) pure and b) Withania somnifera leaf extract incorporated sodium alginate polymer film at different temperatures 

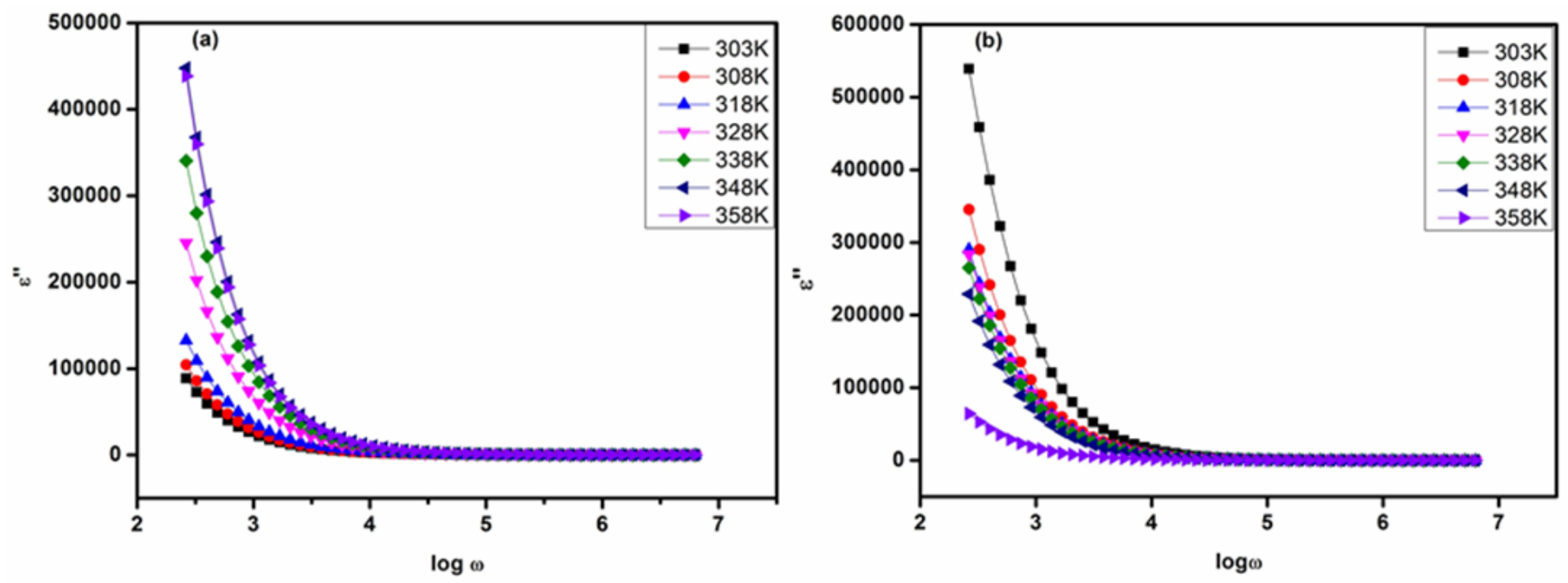

Figure 7

Imaginary part of complex permittivity ( $(")$ of a) pure and b) Withania somnifera leaf extract incorporated sodium alginate film at different temperatures
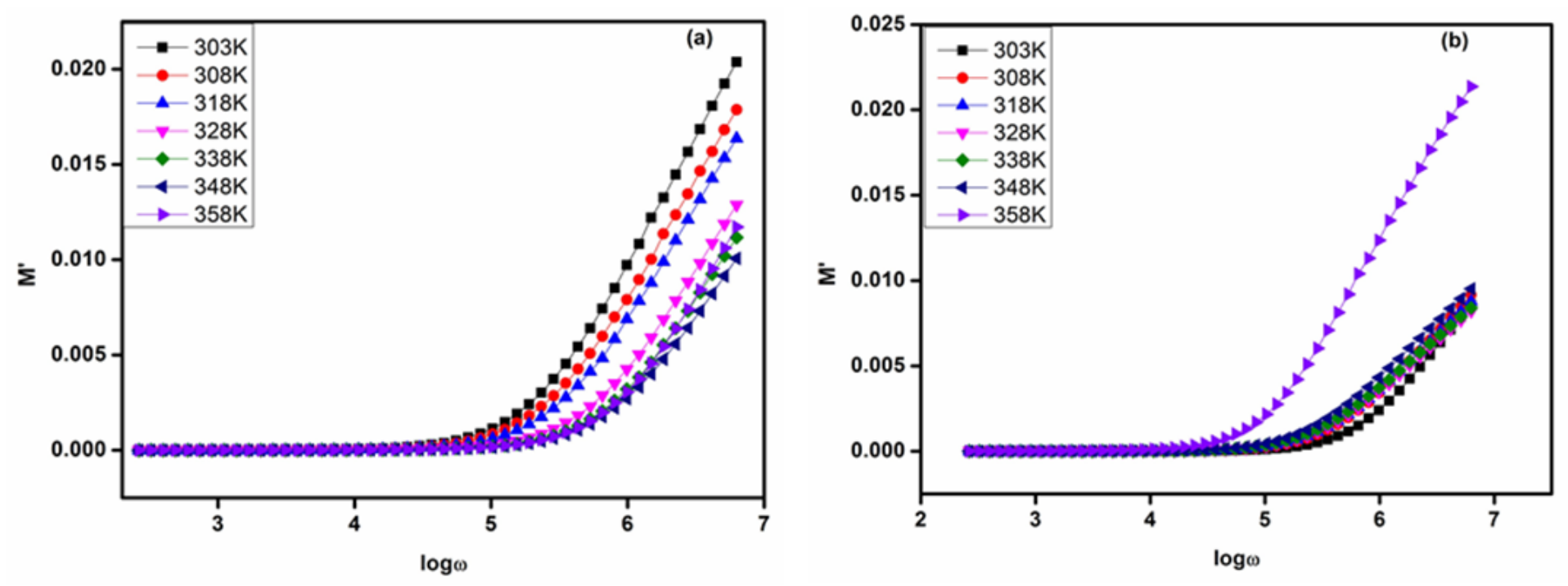

Figure 8

Real part of dielectric modulus ( $\left.M^{\prime}\right)$ of a) Pure and b) Withania somifera leaf extract incorporated sodium alginate polymer film at different temperatures 

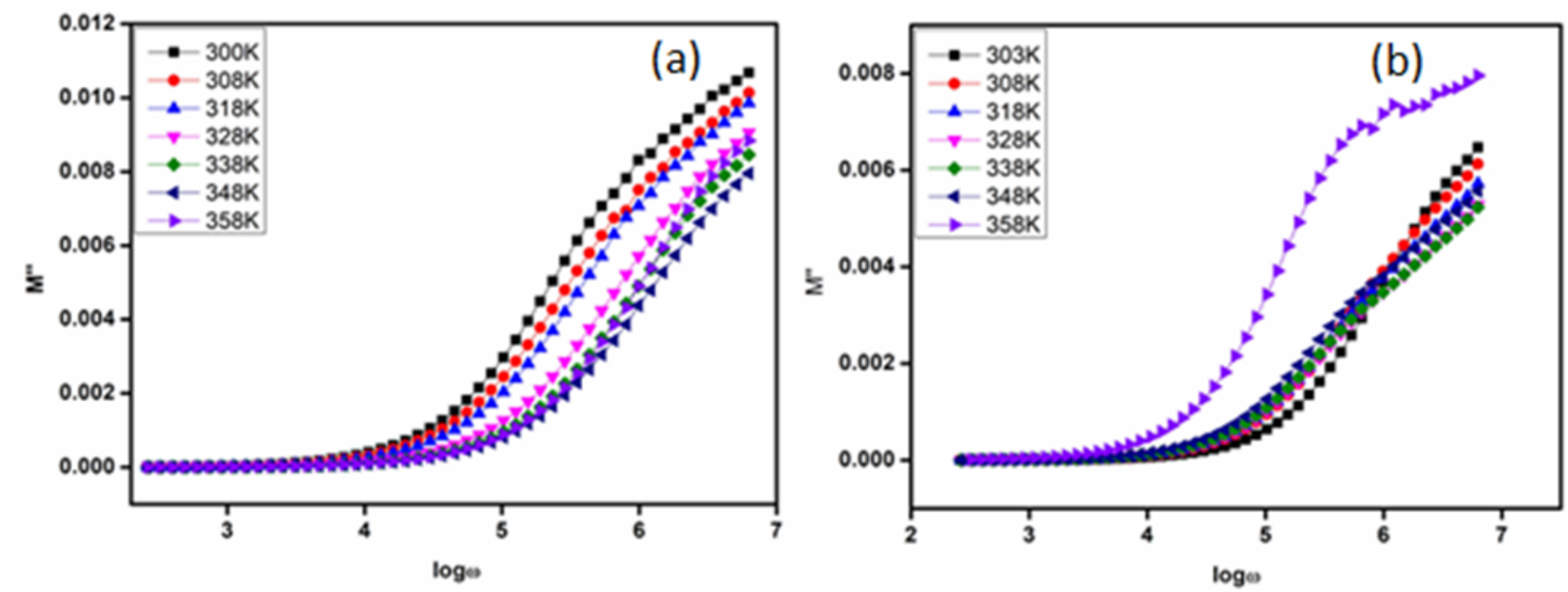

Figure 9

Imaginary part of dielectric modulus ( $\left.\mathrm{M}^{\prime \prime}\right)$ of a) pure and b) Withania somnifera leaf extract incorporated sodium alginate polymer film at different temperatures
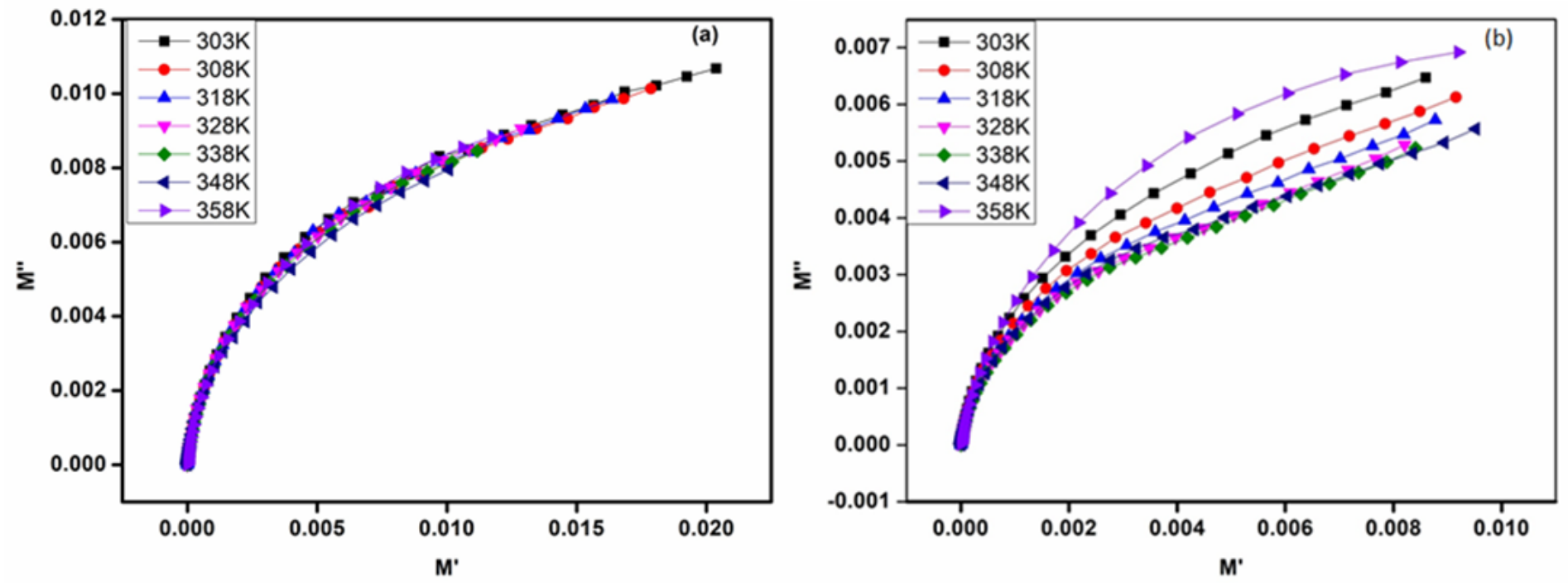

Figure 10

Real ( $\left.M^{\prime}\right)$ Vs imaginary ( $\left.M^{\prime \prime}\right)$ part of dielectric modulus of a) pure and b) Withania somnifera leaf extract incorporated sodium alginate polymer film at different temperatures 

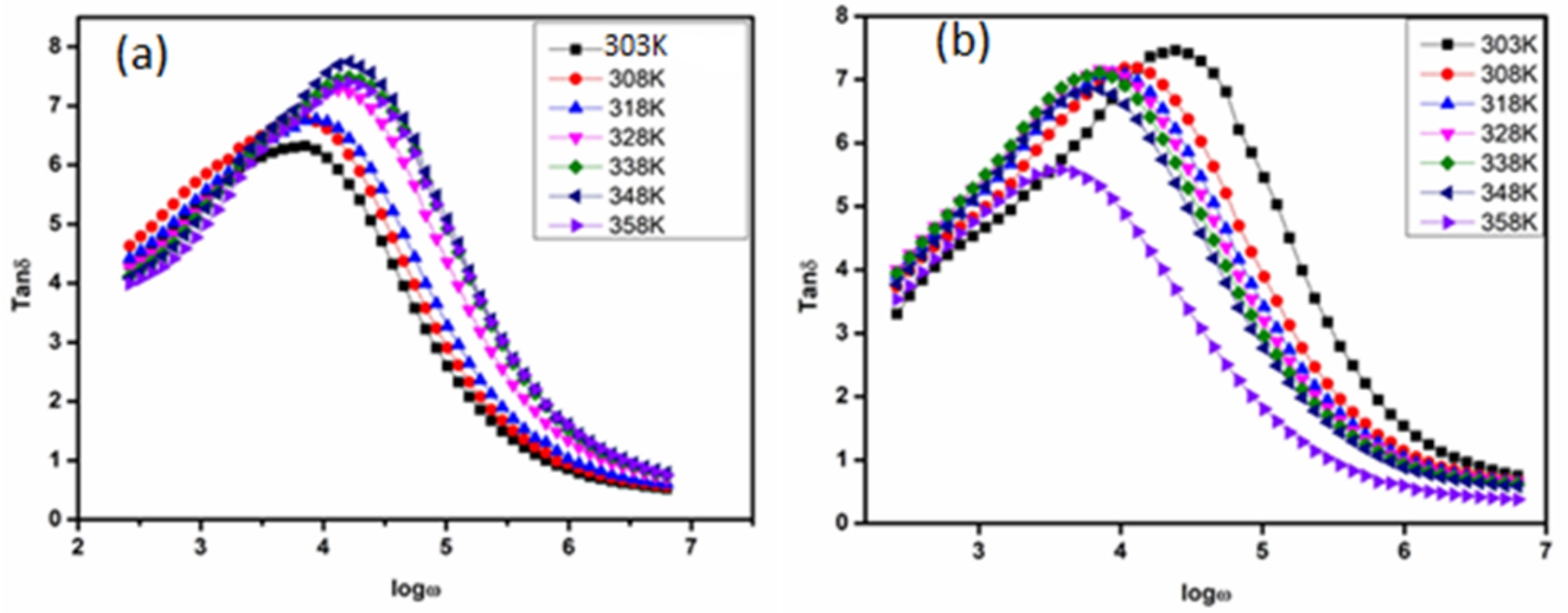

Figure 11

Variation of dielectric energy dissipation factor $\tan \delta$ with frequency of a) pure and b) Withania somnifera leaf extract incorporated sodium alginate polymer film at different temperatures

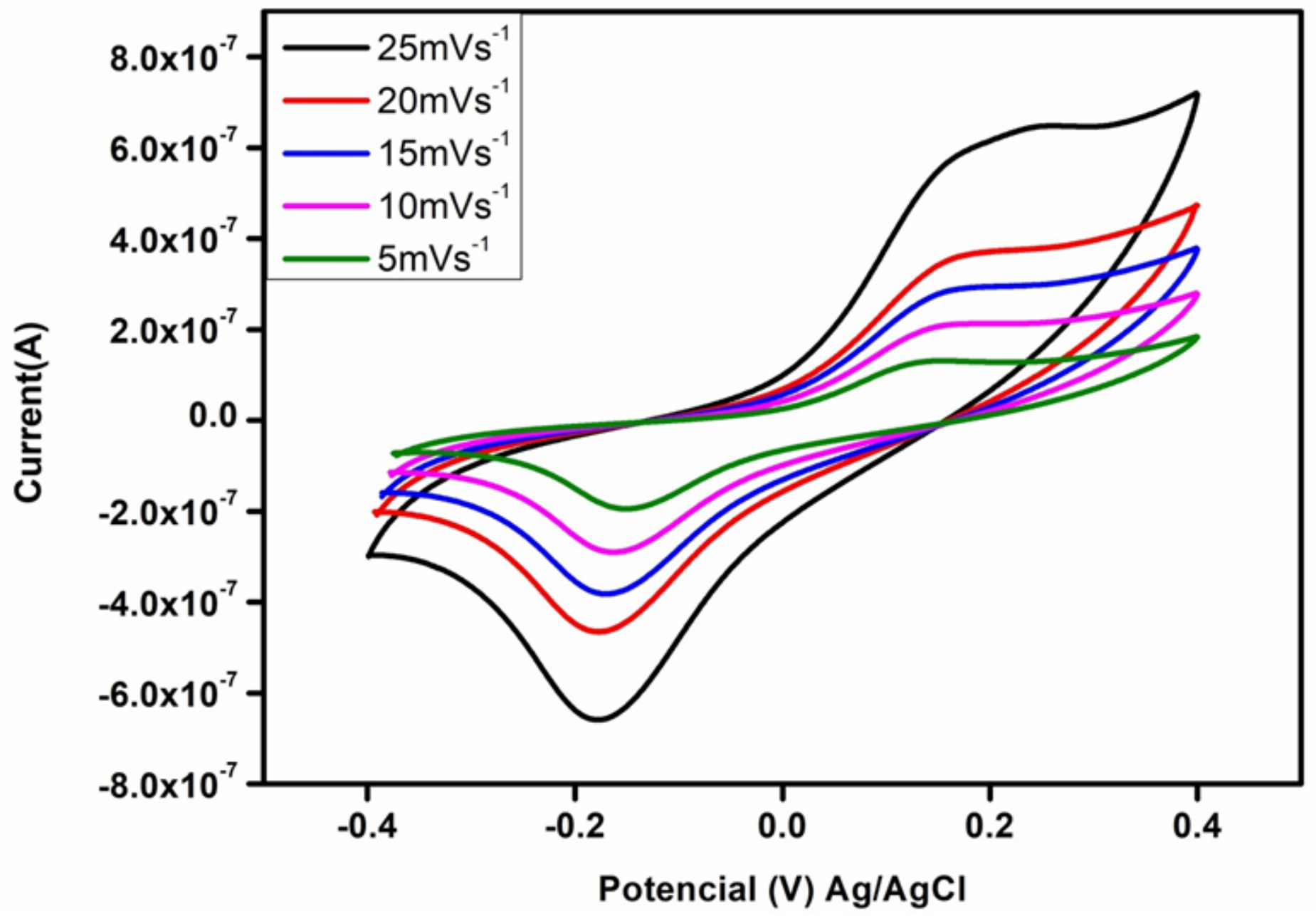

Figure 12 
Cyclic voltammetry of Withania somnifera leaf extract incorporated sodium alginate polymer film at different scan rates 